\title{
A Mouse Model of Postoperative Pain
}

Ashley M. Cowie and Cheryl L. Stucky*

Department of Cell Biology, Neurobiology and Anatomy, Medical College of Wisconsin, Milwaukee, WI 53226, USA

*For correspondence: cstucky@mcw.edu

\begin{abstract}
[Abstract] Postoperative pain is highly debilitating and hinders recovery. Opioids are the main pain medication used for acute postoperative pain. Given the devastating opioid addiction and overdose epidemic across the US, non-opioid pain therapeutics are desperately needed. In order to develop novel, non-opioid therapies for the treatment of postoperative pain and identify the mechanisms underlying this pain, rodent models of incisional pain have been established. The protocol herein describes in detail how to create a mouse model of postoperative pain that was adapted from established protocols. This model of postoperative pain is frequently-used, highly reproducible, and results in peripheral and central nervous system alterations.
\end{abstract}

Keywords: Postoperative pain, Inflammatory pain, Hypersensitivity, Plantar incision, Mouse, Skin and muscle incision

[Background] Postoperative pain is a significant, worldwide problem. Approximately 234.2 million people undergo major surgeries each year (Weiser et al., 2008) and about $80 \%$ of patients experience acute postoperative pain (Gan, 2017). Of these, between $10 \%$ and $50 \%$ of patients, develop chronic pain that continues to severely impact their quality of life (Chapman and Vierck, 2017). One of the factors that are associated with the development chronic postoperative pain, but unlikely the cause, is the severity of acute pain experienced during the first postoperative week (Fletcher et al., 2015; Chapman and Vierck, 2017). Opioids are the main pain medication used for acute postoperative pain (Sen and Bathini, 2015; Tan et al., 2018). Given the opioid epidemic, non-opioid pain therapeutics are needed. Therefore, identifying the mechanisms that underlie acute postoperative pain is necessary for the development of optimal therapies for postoperative pain that may ultimately decrease the severity and/or incidence of chronic postoperative pain. Both rat (Brennan et al., 1996) and mouse (Pogatzki and Raja, 2003) models of acute incisional pain have been developed as preclinical models to identify the molecular, cellular and physiological mechanisms that underlie postoperative pain. However, a detailed description of the mouse model of postoperative pain is lacking. Here we describe in detail a mouse model of postoperative pain that requires incision of both the skin and muscle. Incision of both skin and muscle best mimics invasive surgery that causes intense acute pain and leads to chronic pain (Brennan, 2011; Chapman and Vierck, 2017). Furthermore, incision of skin and muscle ( $\sim 6$ days) creates hypersensitivity that lasts substantially longer than the skin-only ( $\sim 3$ days) incision model ( $\mathrm{Xu}$ and Brennan, 2010). In this protocol, we provide detailed, step-by-step methods adapted from previous protocols (Brennan et al., 1996; Pogatzki and Raja, 2003) for development of a mouse model of 
postoperative pain.

\section{Materials and Reagents}

1. Stainless steel sterile No. 11 surgical blade (World Precision Instruments, Feather Safety Razor Co. Ltd., catalog number: 504170)

2. Sterile 5-0 nylon surgical sutures (AD Surgical, Unify, catalog number: S-N518R13)

3. Surgical tape (3M, Transpore, catalog number: 1527-0)

4. Cotton swab (VWR, Critical Swab, catalog number: 89031-270)

5. Glad Press'n Seal (SAI Infusion Technologies, Glad, catalog number: PSS-70)

6. Sterile nitrile gloves (Kimberly-Clark Professional, Kimtech Pure, catalog number: HC61170)

7. Petri dishes [VWR, 14.5 and 9 663161, Greiner Bio-One, catalog numbers: 82050-912 (small) and 82050-600 (large)]

8. Sterile gauze (Allied Medical, Ardes, catalog number: GA441221)

9. Sharpie extra fine point permanent marker (Staples, Sharpie, catalog number: 37001 )

10. 8-16 week old C57BL/6J mice (JAX, catalog number: 000664)

11. Bacitracin zinc ointment (Fougera Pharmaceuticals Inc, catalog number: 0168-0011-04)

12. Isoflurane (Clipper distributing company LLC., Phoenix, catalog number: 0010250)

13. $75 \%$ ethanol (Fisher Scientific, Decon Laboratories, Inc., catalog number: 22-281-562)

14. Surgical scrub $7.5 \%$ povidone-iodine (Betadine, Veterinary, catalog number: 67618-154-01)

15. Eye lube (Patterson Veterinary, Optixcare Ophthalmic, catalog number: 07-893-2779)

\section{Equipment}

1. $1,000 \mathrm{ml}$ beaker (VWR, PYREX, catalog number: 13912-284)

2. \#55 Dumostar Forceps (Fine Science Tools, Dumont, catalog number: 11295-51)

3. Scalpel handle (Fine Science Tools, catalog number: 10003-12)

4. Iris Forceps, $10 \mathrm{~cm}$, Curved, Serrated (World Precision Instruments, catalog number: 15915)

5. Halsted Mosquito Hemostatic Forceps, $12.5 \mathrm{~cm}$, Straight (World Precision Instruments, catalog number: 15920-G)

6. Vannas Scissors, $8 \mathrm{~cm}$, Curved (World Precision Instruments, catalog number: 14122)

7. Small animal surgery board (Braintree Scientific, Inc., CD+, catalog number: ACD 014)

8. Isoflurane dispenser (Highland Medical Equipment, Drager, catalog number: 16-7001)

9. Sliding top isoflurane induction chamber (Kent Scientific Corporation, catalog number: VetFlo0530LG)

10. Heat Therapy Pump with Pad (Adroit Medical Systems, catalog number: HTP-1500)

11. Isothermal pad (Braintree Scientific, Inc., Deltaphase, catalog number: DPIP)

12. Digital calipers (VWR, catalog number: 62379-531)

13. Steri 250 Bead Sterilizer Bead Bath (Lab Unlimited, Simon Keller Ltd., catalog number: 4AJ- 
6286283)

14. Microwave (Emerson, $1,000 \mathrm{~W}$, catalog number: B007Q45CIS)

15. Home cage containing Aspen Sani Chips ${ }^{\circledR}$ (P.J. Murphy Forest and Products, Sani Chips ${ }^{\circledR}$ )

\section{Software}

\section{GraphPad Prism 7}

\section{Procedure}

1. Sterilize surgical area with ethanol and sterilize tools in bead bath of at least $325^{\circ} \mathrm{C}$ for $30 \mathrm{~s}$. Rest sterile tools, sutures, and blade on sterile gauze.

2. Warm Heat Therapy Pump pad to $41^{\circ} \mathrm{C}$ and warm the Isothermal pad in microwave for $1 \mathrm{~min}$. Place a new, clean home cage on the Heat Therapy Pump pad. Place a laboratory paper towel over the warm Isothermal pad and cover with sterile Press'n Seal and surgery board.

3. To induce anesthesia, put the mouse in the beaker chamber with paper towel on lid containing $1 \mathrm{ml}$ of isoflurane (Figure 1) and wait for approximately $30 \mathrm{~s}$ or until the mouse can no longer right itself. Alternatively, an isoflurane induction chamber that is included with the isoflurane dispenser can be used to induce anesthesia.

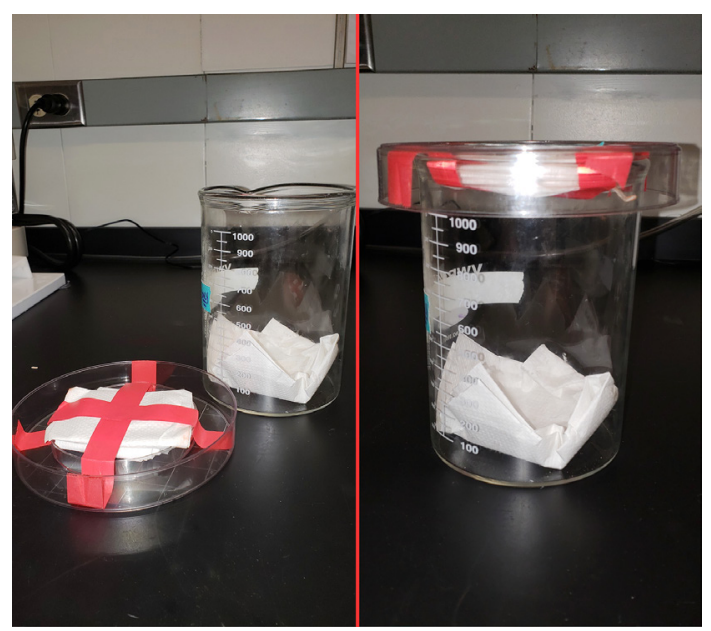

Figure 1. Brief inhalational anesthesia. The mouse was briefly anesthetized by inhalation in chamber with $1 \mathrm{ml}$ isoflurane.

4. Quickly remove the mouse from the isoflurane chamber and apply eye lube using a cotton swab to each eye. Further anesthetize the mouse with $1.5 \%-2 \%$ inhaled isoflurane by placing the head of the mouse into the nose cone attached to the isoflurane dispenser and cover the mouse (except the hindpaw designated for operation) with sterile Press'n Seal (Figure 2). 


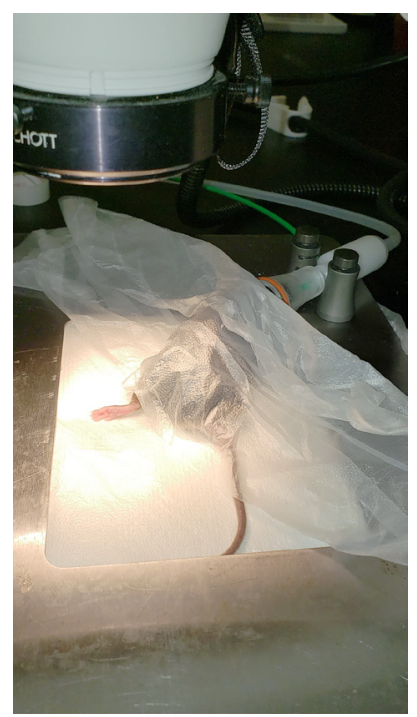

Figure 2. Continuous inhalational anesthesia. The mouse was continuously anesthetized through a nose cone with $1.5 \%-2 \%$ isoflurane.

For Steps 5-13 also see Video 1.

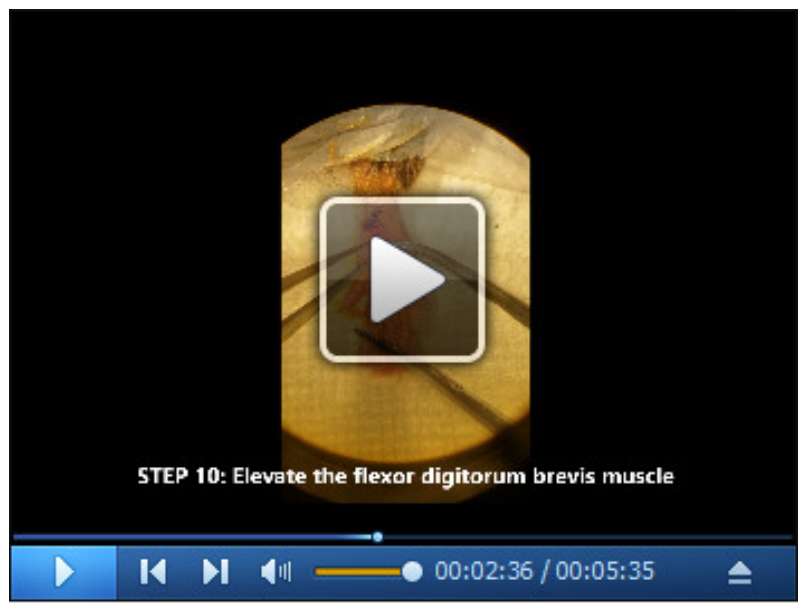

Video 1. Plantar incision surgery. This video shows how the model of postoperative pain is made by making an incision through the plantar skin and flexor digitorum brevis muscle (This video was made at the Medical College of Wisconsin and was performed according to guidelines on Animal Care and approved by the Animal Research Ethics Board of the Medical College of Wisconsin under protocol \#0383).

5. Adhere the hindpaw to the surface by taping the toes down with surgical tape (Figure 3). 


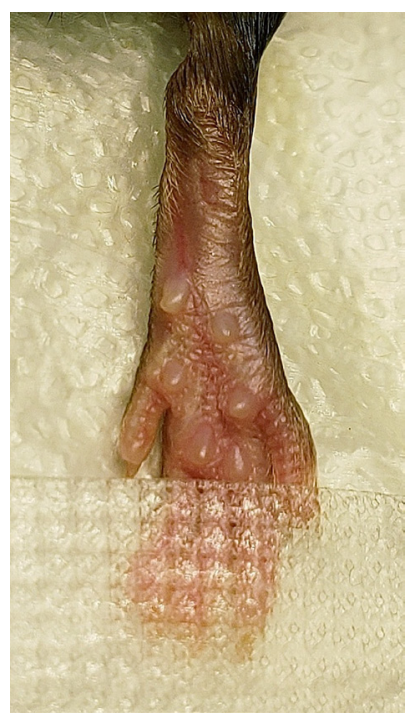

Figure 3. Securing the hindpaw to surgical surface. The hindpaw was secured to the surface using surgical tape.

6. Once the hindpaw is secured, apply a cotton swab of $75 \%$ ethanol followed by a new cotton swab of betadine. Repeat for a total of 3 applications each.

7. Measure $2 \mathrm{~mm}$ from the proximal edge of heel using a digital caliper and place a dot with a permanent marker at this location in the middle of the hindpaw. From the first dot, measure 5 $\mathrm{mm}$ towards the toes down the center of the hindpaw and place a second dot (Figure 4).

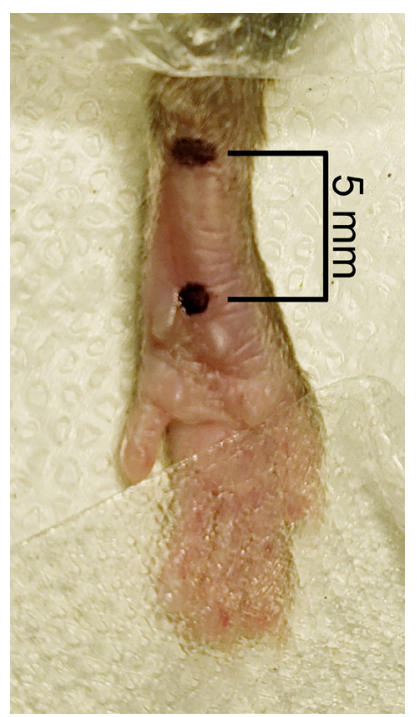

Figure 4. Measurement of incision. Two dots were placed in the middle of the hindpaw, one $2 \mathrm{~mm}$ from the heel and the second $5 \mathrm{~mm}$ from the first dot.

8. Check that the mouse is fully anesthetized by lightly pinching the most medial toe (most likely this toe could not be secured by the surgical tape) with the forceps. If the mouse flinches, wait until the mouse no longer reacts to the toe pinch before proceeding to Step 9. 
9. Stabilize the hindpaw by placing the forceps on each side of the heel and make a longitudinal incision through the skin and fascia from the first dot to the second dot (Figure 5).

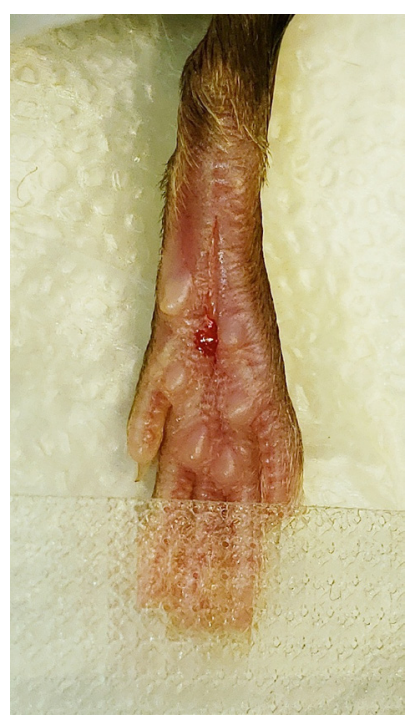

Figure 5. Cutaneous incision. A $5 \mathrm{~mm}$ longitudinal incision was made with a No. 11 scalpel.

10. Spread the skin away from the flexor digitorum brevis muscle with the forceps. Elevate the flexor digitorum brevis muscle by inserting one end of the curved forceps underneath the lateral edge of the flexor digitorum brevis muscle and pushing the forceps through to the medial side of the muscle (Figure 6).

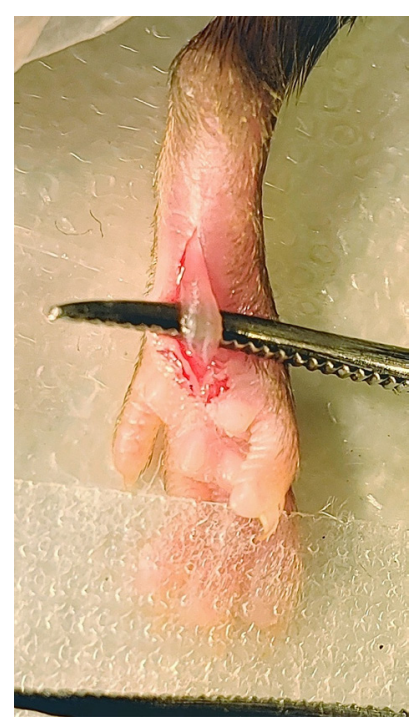

Figure 6. Elevation of flexor digitorum brevis muscle. Curved forceps were inserted under the flexor digitorum brevis muscle to elevate the muscle. 
11. Make a longitudinal incision with the scalpel through the entire belly of the muscle from the origin and insertion taking care not to sever the muscle completely from the origin and insertion, making sure to cut the belly of the muscle into two halves (Figure 7).

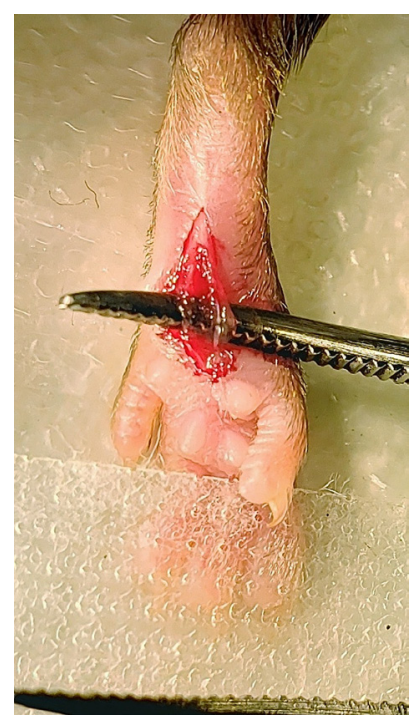

Figure 7. Muscle incision. A longitudinal incision was made through the muscle belly of the elevated flexor digitorum brevis muscle from proximal to distal ends of the cutaneous incision.

12. To suture the wound, remove the curved forceps from underneath the muscle and elevate the edges of the skin surrounding the wound with forceps. Close the wound by putting two sutures in the skin (but not muscle) approximately $2 \mathrm{~mm}$ apart using 5-0 nylon sutures and a hemostat (Figure 8).

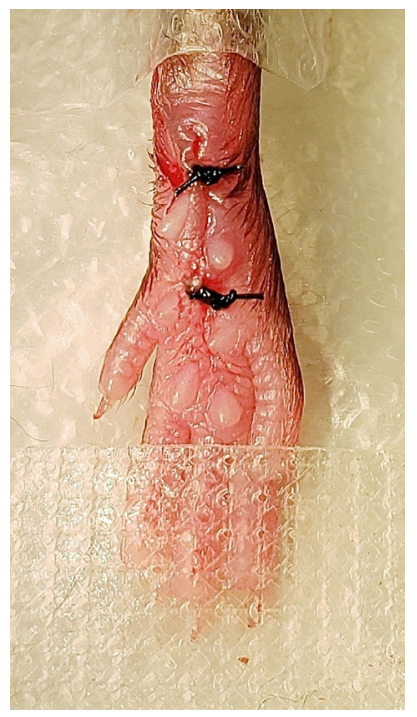

Figure 8. Cutaneous suturing. The skin was closed with two 5-0 nylon sutures. 
13. Apply a generous amount of bacitracin ointment to the wound using a cotton swab and place the mouse in the new cage located on the Heat Therapy Pump pad from Step 2.

\section{Data analysis}

Statistical significance was determined with GraphPad Prism 7 Software and graphs are shown as mean \pm SEM. A two-way ANOVA with a Sidak post-hoc was used to determine statistical significance for mechanical and heat thresholds. A complete description of statistics used for analyzing mechanical and heat threshold behavioral data is provided in Cowie et al. (2018).

\section{Notes}

1. It is important to perform the operation as quickly as possible to reduce the need for repeated anesthesia and any side effects from anesthesia. In our laboratory, it takes a trained surgeon approximately $5 \mathrm{~min}$ to perform this procedure.

2. For a control, a sham surgery is performed by anesthetizing the mouse, sterilizing the hindpaw as in Step 5, and applying bacitracin ointment to the plantar hindpaw.

3. If bleeding occurs during the procedure, apply pressure to the incision site with a cotton swab until the bleeding stops.

4. Mice are housed together.

5. No care is required after surgery except for monitoring of sutures.

6. If using mice past postoperative day 3 , remove sutures on postoperative day 4 . Mice that pulled out sutures before postoperative day 2 must be removed from the study due to poor wound closure.

7. For consistent behavioral results, apply mechanical and heat stimuli to the medial-posterior aspect of the plantar hindpaw (Figure 9). This area is the least sensitive at baseline because the heel is weight bearing whereas other areas near the pads are more sensitive and variable in withdrawal threshold. Therefore, using the heel area that provides a consistently high baseline withdrawal threshold allows for the best detection of change due to incision (Brennan et al., 1996). Mice were acclimated for $1 \mathrm{~h}$ in Plexiglass chambers placed on either a mesh platform (mechanical threshold) or glass platform (heat threshold). Calibrated von Frey monofilaments ( 0.09 to $19.6 \mathrm{mN}$ ) were applied to the plantar hindpaw and the withdrawal threshold for each animal was calculated using the up-down method (Dixon, 1980; Chaplan et al., 1994). The Hargreaves assay was used to measure heat sensitivity as previously established (Hargreaves et al., 1988; Jackson et al., 1995; Barabas and Stucky, 2013; Cowie et al., 2018). Withdrawal latencies to a focused radiant heat source (IITC, Life Sciences Instruments) underneath the glass platform were measured 3 times and averaged for each mouse. A cutoff of $20 \mathrm{~s}$ was used to avoid injury. Examples of mechanical and heat hypersensitivity following incision are shown in Figure 10. 


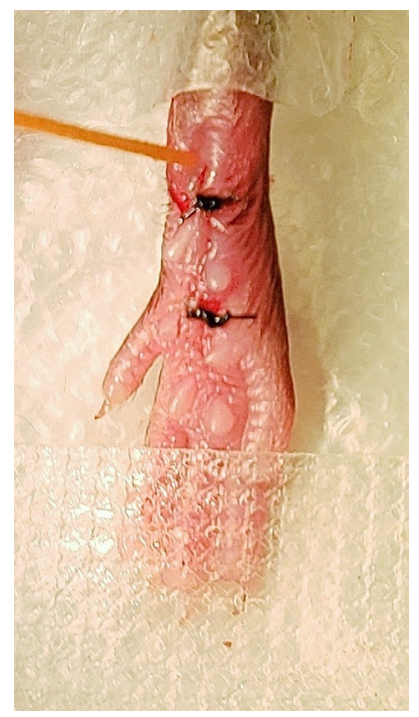

Figure 9. Application of von Frey monofilament. An orange von Frey monofilament was applied to most sensitive location following incision.

A

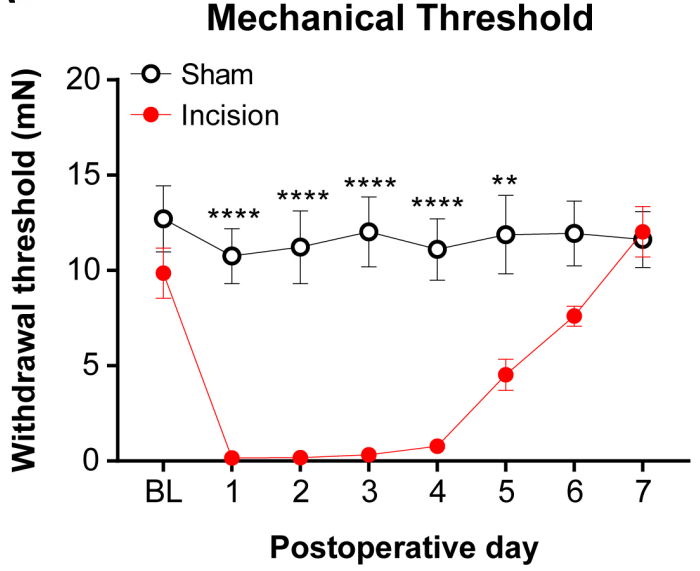

B

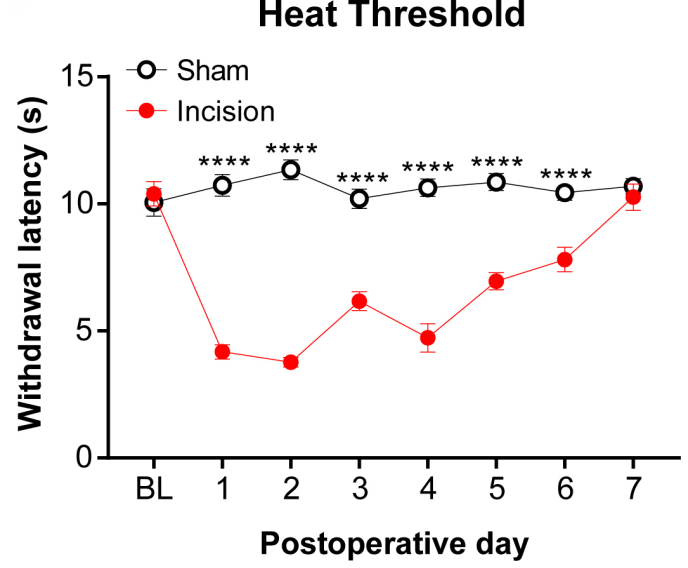

Figure 10. Mechanical and heat thresholds following incision. A. von Frey monofilaments was applied to most sensitive location following incision and the Dixon up-down method (Dixon, 1980) was used to determine mechanical threshold. B. The Hargreaves assay (Hargreaves et al., 1988) was used to measure the withdrawal threshold in response to a radiant heat source that was applied to the most sensitive location following incision. These data were modified from Cowie et al. (2018). Data shown as mean \pm SEM, repeated-measures two-way ANOVA and Sidak post-hoc analysis, ${ }^{* *} P<0.01$ and ${ }^{* * *} P<0.0001$ sham versus incision. For $(A)$ and $(B)$, $\mathrm{n}=8$ male mice per group. 


\section{Acknowledgments}

This protocol was adapted from established published procedures (Brennan et al., 1996; Pogatzki and Raja, 2003). This work was supported by the National Institute of Neurological Disorders and Stroke grants NS040538 and NS070711 to C.L.S and F31GM123778 to A.M.C. The Research and Education Component of the Advancing a Healthier Wisconsin Endowment at the Medical College of Wisconsin provided partial support. The authors thank Timothy J Brennan, Ph.D., MD for his review of the manuscript.

\section{Competing interests}

The authors declare no competing financial or non-financial interests.

\section{Ethics}

All animal procedures were carried out in accordance with the National Institute of Health guidelines and approved by the Institutional Animal Care and Use Committee of the Medical College of Wisconsin (AUA\#0383).

\section{References}

1. Barabas, M. E. and Stucky, C. L. (2013). TRPV1, but not TRPA1, in primary sensory neurons contributes to cutaneous incision-mediated hypersensitivity. Mol Pain 9: 9.

2. Brennan, T. J. (2011). Pathophysiology of postoperative pain. Pain 152(3 Suppl): S33-40.

3. Brennan, T. J., Vandermeulen, E. P. and Gebhart, G. F. (1996). Characterization of a rat model of incisional pain. Pain 64(3): 493-501.

4. Chaplan, S. R., Bach, F. W., Pogrel, J. W., Chung, J. M. and Yaksh, T. L. (1994). Quantitative assessment of tactile allodynia in the rat paw. J Neurosci Methods 53(1): 55-63.

5. Chapman, C. R. and Vierck, C. J. (2017). The transition of acute postoperative pain to chronic pain: An integrative overview of research on mechanisms. J Pain 18(4): 359 e1-359 e38.

6. Cowie, A. M., Moehring, F., O'Hara, C. and Stucky, C. L. (2018). Optogenetic inhibition of CGRPa sensory neurons reveals their distinct roles in neuropathic and incisional pain. $J$ Neurosci 38(25): 5807-5825.

7. Dixon, W. J. (1980). Efficient analysis of experimental observations. Annu Rev Pharmacol Toxicol 20: 441-462.

8. Fletcher, D., Stamer, U. M., Pogatzki-Zahn, E., Zaslansky, R., Tanase, N. V., Perruchoud, C., Kranke, P., Komann, M., Lehman, T. and Meissner, W. (2015). Chronic postsurgical pain in Europe: An observational study. Eur J Anaesthesiol 32(10): 725-734. 
9. Gan, T. J. (2017). Poorly controlled postoperative pain: prevalence, consequences, and prevention. J Pain Res 10: 2287-2298.

10. Hargreaves, K., Dubner, R., Brown, F., Flores, C. and Joris, J. (1988). A new and sensitive method for measuring thermal nociception in cutaneous hyperalgesia. Pain 32(1): 77-88.

11. Jackson, D. L., Graff, C. B., Richardson, J. D. and Hargreaves, K. M. (1995). Glutamate participates in the peripheral modulation of thermal hyperalgesia in rats. Eur J Pharmacol 284(3): 321-325.

12. Pogatzki, E. M. and Raja, S. N. (2003). A mouse model of incisional pain. Anesthesiology 99(4): 1023-1027.

13. Sen, S. and Bathini, P. (2015). Auditing analgesic use in post-operative setting in a teaching hospital. J Clin Diagn Res 9(4): FC01-04.

14. Tan, W. H., Yu, J., Feaman, S., McAllister, J. M., Kahan, L. G., Quasebarth, M. A., Blatnik, J. A., Eagon, J. C., Awad, M. M. and Brunt, L. M. (2018). Opioid medication use in the surgical patient: An assessment of prescribing patterns and use. J Am Coll Surg 227(2): 203-211.

15. Weiser, T. G., Regenbogen, S. E., Thompson, K. D., Haynes, A. B., Lipsitz, S. R., Berry, W. R. and Gawande, A. A. (2008). An estimation of the global volume of surgery: a modelling strategy based on available data. Lancet 372(9633): 139-144.

16. Xu, J. and Brennan, T. J. (2010). Guarding pain and spontaneous activity of nociceptors after skin versus skin plus deep tissue incision. Anesthesiology 112(1): 153-164. 